\title{
Jogos Digitais para Ensino e Aprendizagem de Programação: uma Revisão Sistemática da Literatura
}

\author{
Thiago Reis da Silva ${ }^{1}$, Taina Jesus Medeiros ${ }^{1}$, Eduardo Henrique da S. Aranha ${ }^{1}$ \\ ${ }^{1}$ Programa de Pós-Graduação em Sistemas Computacionais - PPgSC \\ Universidade Federal do Rio Grande do Norte - UFRN \\ Caixa Postal 1524 - Campus Universitário Lagoa Nova - CEP 59072-970 - Natal/RN - \\ Brasil \\ \{trsilva.si, tainajmedeiros\}@gmail.com, eduardoaranha@dimap.ufrn.br
}

\begin{abstract}
Introduction: teaching programming is a difficult task due to the complex nature of the subject, the negative stereotypes associated with it and the failure of programming courses to encourage student understanding. Objectives: To investigate the efficacy of using digital games as a tool to assist in the process of teaching and learning programming. Method: A systematic literature review was performed to integrate the results from several previously published studies. Results: Among 6,518 primary studies, 29 were included in this study, 97\% of these studies report that the use of games is an effective tool for teaching and learning programming.
\end{abstract}

Resumo. Introdução: ensino de programação é uma tarefa difícil, devido à natureza complexa do assunto, e também dos estereótipos negativos que estão associados e porque as disciplinas de programação muitas vezes não conseguem estimular a compreensão do aluno. Objetivos: investigar a eficácia do uso de jogos digitais como ferramenta para auxiliar o processo de ensino e aprendizagem de programação. Método: foi realizada uma revisão sistemática da literatura, para integrar os resultados oriundos de diversos estudos publicados anteriormente. Resultados: dos 6518 estudos primários, 29 foram incluidos nesse estudo, destes 97\% dos estudos relatam que a utilização de jogos é uma ferramenta eficaz de ensino e aprendizagem de programação.

\section{Introdução}

Várias são as dificuldades enfrentadas pelos alunos iniciantes durante o processo de aprendizagem de programação. Existem várias possibilidades de origem destas dificuldades, como destaca Raabe e Silva (2005), seja pela exigência lógico-matemático predominante na disciplina, ou até mesmo pelo ritmo de aprendizagem de cada aluno. Aprender programar tem sido destacado pelos iniciantes como uma tarefa difícil e complexa. Devido às dificuldades continuamente enfrentadas por alunos iniciantes em cursos das áreas tecnológicas, tem resultado em altas taxas de evasão e desistência [Adamo-Villani, Haley-Hermiz e Cutler 2013].

Vários esforços têm sido feitos pelos pesquisadores e educadores para tentar superar as dificuldades encontradas pelos iniciantes nesta área. Tais tentativas são muitas vezes referidas como intervenções ou métodos. Um método que vem sendo explorado como ferramenta para auxiliar o ensino e aprendizagem de programação é o 
uso de jogos digitais [Rebouças et. al. 2010] [Marques et. al. 2011]. Sá, Teixeira e Fernandes (2007) destaca que o uso de jogos digitais nas atividades de ensino possibilita oferecer ao aprendiz momentos lúdicos e interativos como etapas do processo de aprendizagem.

Neste contexto, o objetivo deste trabalho foi o desenvolvimento de uma Revisão Sistemática da Literatura (RSL), a fim de investigar a eficácia da utilização de jogos digitais como ferramenta para auxiliar o processo de ensino e aprendizagem de programação. Uma RSL é uma pesquisa em profundidade de um fenômeno de interesse que produz resultados específicos e detalhados por meio da análise de conteúdo e qualidade do material pesquisado [Kitchenham et. al. 2009]. A RSL foi utilizada visando extrair, catalogar, analisar e sintetizar dados de vários artigos publicados em congressos, jornais e periódicos internacionais, que abordem a temática desta revisão.

Portanto, este artigo segue organizado em cinco seções, a partir desta introdução. A Seção 2 apresenta o método de pesquisa utilizado. Na Seção 3 a análise desta revisão. Logo após, na Seção 4, os resultados gerais e, por fim, na Seção 5 são feitas as considerações finais.

\section{Método utilizado}

Esta RSL está estruturada com base nas diretrizes originais propostas por Kitchenham et. al. (2009). No caso desta revisão o objetivo foi determinar a eficácia do uso de jogos digitais como ferramenta de ensino e aprendizagem de programação, e também fazer uma análise do panorama das pesquisas e experiências práticas em relação ao uso de jogos digitais para o processo de ensino e aprendizagem de programação no contexto internacional entre os anos de 2009 a 2013. Foram incluídos artigos de 1 de janeiro de 2009 a 31 de dezembro de 2013.

A RSL realizada neste trabalho visa a responder às questões apresentadas na Tabela 1. A primeira coluna desta tabela apresenta o código da questão de pesquisa, e a segunda, a descrição da mesma.

Tabela 1: Questões de Pesquisa.

\begin{tabular}{|c|l|}
\hline Questões & \multicolumn{1}{|c|}{ Descrição } \\
\hline QP1 & $\begin{array}{l}\text { Que linguagens de programação estão sendo ensinados pelos estudos que fazem uso de } \\
\text { jogos como método de ensino? }\end{array}$ \\
\hline QP2 & $\begin{array}{l}\text { Que tipos de estudos estão sendo realizados por pesquisadores que investigam o ensino de } \\
\text { programação utilizando jogos? }\end{array}$ \\
\hline QP3 & $\begin{array}{l}\text { Qual é a escala (por exemplo, número de participantes) dos estudos que estão sendo } \\
\text { realizados por pesquisadores? }\end{array}$ \\
\hline QP4 & Os estudos reportados indicam que o uso de jogos para ensinar programação é eficaz? \\
\hline QP5 & Quais os níveis de escolaridade estão sendo destinados os estudos? \\
\hline
\end{tabular}

\subsection{Processo de busca, critérios de inclusão e exclusão}

O processo de busca de estudos primários consistiu em: i) utilizar os engenhos de buscas, IEEE Xplorer, ACM Digital Library e ScienceDirect, sendo identificados como potencialmente útil por estudos anteriores [Major, Kyriacou e Brereton 2011]; ii) realizar buscas manuais nos proceedings do Technical Symposium on Computer Science Education (SIGCSE), Conference on International Computing Education Research (ICER), Conference on Foundations of Digital Games (FDG), Transactions 
on Computing Education (TOCE), Conference on Innovation and Technology in Computer Science Education (ITiCSE), International Conference on Digital Game and Intelligent Toy Enhanced Learning (DIGITEL), Workshop on Network and System Support for Games (NetGames), Symposium on Computational Intelligence and Games (CIG), Conference on Information Technology Education (SIGITE), Symposium on Interactive 3D Graphics and Games (I3D), International Conference on Software Engineering (ICSE), International Symposium on Empirical Software Engineering and Measurement (ESEM) e International Conference on Evaluation and Assessment in Software Engineering (EASE); e iii) realizar buscas no site dos Periódicos Journal Computer and Education (JCE), Journal of Computing Sciences in Colleges (JCSC) e International Journal of Educational Research (IJER), utilizando o mecanismo de busca próprio deste site. Os resultados deste processo de busca podem ser visualizados em: $<$ http://goo.gl/u6idfK $>$.

Uma abordagem em três etapas foi utilizada no processo de busca. Na primeira etapa, todos os estudos primários recuperados foram avaliados a fim de se identificar aqueles relevantes para responder às questões de pesquisa, um total de 6518 estudos foram analisados. Após a leitura dos títulos, resumos e palavras chaves, este conjunto inicial foi reduzido para 95. Durante esta triagem, foram aplicados Critérios de Inclusão (CI) e Critérios de Exclusão (CE) para cada estudo recuperado (ver Tabela 2).

Tabela 2: Critérios de inclusão e exclusão.

\begin{tabular}{|c|c|}
\hline Critérios de Inclusão & Critérios de Exclusão \\
\hline $\begin{array}{l}\text { - CI1: Publicações que relataram o uso dos jogos } \\
\text { no ensino de programação para os alunos de } \\
\text { ensino básico, técnico e superior; } \\
\text { - CI2: No caso de jornais ou conferências } \\
\text { relataram o mesmo estudo apenas o artigo mais } \\
\text { recente foi incluído; e } \\
\text { - CI3: Publicações entre } 2009 \text { a } 2013 \text {. }\end{array}$ & $\begin{array}{l}\text {-CE1: Publicações foram excluídas se seu foco } \\
\text { principal não foi sobre o uso dos jogos para o } \\
\text { ensino de programação; } \\
\text {-CE2: Artigos que apenas propõem uma } \\
\text { abordagem ou descrevem o uso de jogos para } \\
\text { ensinar programação, sem obtenção de resultados; } \\
\text {-CE3: Documentos que estão disponíveis na forma } \\
\text { de resumos ou apresentações e estudos secundários } \\
\text { (ou seja, revisões sistemáticas da literatura e } \\
\text { mapeamentos sistemáticos); } \\
\text { •CE4: Publicações foram excluídas se eles não são } \\
\text { escritos em Inglês; e } \\
\text {-CE5: Artigos duplicados. }\end{array}$ \\
\hline
\end{tabular}

Em uma segunda etapa, procedeu-se à leitura dos resumos e conclusão e novamente à aplicação dos CI e CE dos 95 artigos candidatos e o resultado foi um subconjunto de 29 estudos primários. É importante ressaltar que, em alguns casos, a leitura de outras partes dos artigos foi realizada para se proceder à seleção. Na terceira etapa, os 29 estudos que compõe a seleção final foram lidos na íntegra.

\subsection{String de busca}

As palavras-chaves utilizadas na string de busca para obtenção dos estudos primários deste RSL são: Game, Teaching e Programming. Com base neste conjunto de palavraschave, a string de busca adequada foi gerada (Tabela 3). É importante salientar que, apesar de não ter sido apresentado na Tabela 3, teve-se o cuidado de incluir na string os termos variantes (exemplos, education, code, program) e outras flexões gramaticais.

Tabela 3: String de busca. 


\begin{tabular}{|c|c|c|c|}
\hline \multirow{2}{*}{ String } & \multicolumn{3}{|c|}{ Engenhos de Busca } \\
\cline { 2 - 4 } & IEEE & ACM & ScienceDirect \\
\hline teaching AND programming AND games & 147 & 212 & 213 \\
\hline
\end{tabular}

\subsection{Avaliação da qualidade}

Cada publicação foi avaliada pela sua qualidade. Este processo de avaliação da qualidade foi realizado durante a fase de extração de dados e garantiu que estudos incluídos tivessem uma contribuição valiosa para a RSL. Os 11 critérios de avaliação (Tabela 4) de qualidade utilizados são discutidos por Dyba e Dingsøyr (2008).

Tabela 4: Critérios de Avaliação.

\begin{tabular}{|c|l|}
\hline Critérios & \multicolumn{1}{c|}{ Descrição } \\
\hline QA1 & $\begin{array}{l}\text { O estudo está baseado em pesquisas empíricas ou em relatos de experiência com base em } \\
\text { relatórios ou na opinião de especialistas? }\end{array}$ \\
\hline QA2 & Existe uma definição clara dos objetivos da pesquisa? \\
\hline QA3 & Existe uma descrição adequada do contexto em que a pesquisa foi realizada? \\
\hline QA4 & O planejamento da pesquisa foi adequado para abordar os objetivos da pesquisa? \\
\hline QA5 & A estratégia de extração de dados foi adequada aos objetivos da pesquisa? \\
\hline QA6 & Havia um grupo de controle com o qual pudesse comparar tratamentos? \\
\hline QA7 & Os dados foram coletados de forma que abordasse as questões de pesquisa? \\
\hline QA8 & A análise dos dados foi suficientemente rigorosa? \\
\hline QA9 & Será que a relação entre pesquisador e participantes foi considerada um grau adequado? \\
\hline QA10 & Existe uma indicação clara dos resultados? \\
\hline QA11 & É o estudo de valor para pesquisa ou prática? \\
\hline
\end{tabular}

Os dois primeiros critérios foram utilizados para excluir estudos que não indicam claramente os objetivos da pesquisa. Isto representa o limiar mínimo de qualidade que foi observada durante esta RSL. Os critérios restantes visam determinar o rigor, análise e credibilidade dos métodos de pesquisa empregados, bem como a relevância e a qualidade de cada estudo para esta revisão. As respostas para cada questão foram tabuladas e atribuídas um valor de 1 ("Sim") ou 0 ("Não"). A fim de testar a validade do procedimento de avaliação de qualidade um segundo e terceiro revisor, foi dada as mesmas amostras encontradas dos artigos e foi convidado a avaliar a sua qualidade com base nos mesmos critérios de avaliação de qualidade definidos. Não houve discordância sobre a avaliação da qualidade desses trabalhos.

\subsection{Extração dos dados a partir dos estudos primários selecionados}

Nesta fase, foram extraídos os seguintes dados sobre os estudos selecionados: objetivos do estudo; método do estudo (por exemplo, experimento controlado, relato de experiência, estudo de caso, entre outros); número de participantes envolvidos; como os dados foram coletados e analisados durante o estudo; escolaridade a qual a pesquisa foi aplicada (por exemplo, ensino médio ou superior); tipo de linguagem de programação que está sendo ensinado (por exemplo, Java, C, dentre outras); os resultados, conclusões e dificuldades encontradas; eficácia do uso de jogos como um método para ensinar programação; e a avaliação de qualidade do estudo. Todos os dados foram extraídos por um revisor, enquanto o segundo e terceiro revisor, analisou o resultado geral dos estudos. Estes resultados foram então comparados. Como não existiram anomalias significativas ficou evidente que a partir desta atividade de validação da estratégia de extração de dados foi considerado apropriado. Todos os dados extraídos foram armazenados em uma planilha. 


\section{Análise}

Como resultado de busca baseadas em títulos, resumos e palavras-chaves, de um total de 6518 artigos, foram pré-selecionados 95 estudos. Estes 95 estudos foram analisados também os resumos e as conclusões e aplicados os critérios de inclusão e exclusão. Depois dessa segunda análise, foram excluídos 66 artigos, restando 29 artigos relevantes, selecionados para a extração de dados. As referências bibliográficas destes artigos podem ser visualizadas em: <goo.gl/zXIbqj $>$. Desses 66 artigos excluídos, 1 artigo era duplicado, 63 baseado no critério de exclusão $\mathrm{C} 2,1$ baseado no critério de exclusão $\mathrm{C} 3$ e outro tomado como base o critério $\mathrm{C} 5$.

\subsection{Avaliação da qualidade dos estudos reportados}

Os resultados desta avaliação da qualidade são apresentados na Tabela 5. Todos os artigos incluídos na revisão foram baseados em pesquisas empíricas ou apresentando relatos de experiências (QA1), e que os objetivos estivessem claramente definidos (QA2). Dos 29 estudos, 18 ofereceu alguma descrição do contexto em que a pesquisa foi realizada (QA3), enquanto 26 foram considerados como tendo um projeto de pesquisa apropriado (QA4).

Tabela 5: Resultados da Avaliação da Qualidade.

\begin{tabular}{|c|c|c|c|c|c|c|c|c|c|c|c|c|}
\hline Estudos & QA1 & QA2 & QA3 & QA4 & QA5 & QA6 & QA7 & QA8 & QA9 & QA10 & Q11 & Total \\
\hline E1 & 1 & 1 & 0 & 1 & 1 & 0 & 0 & 0 & 0 & 1 & 1 & 6 \\
\hline E2 & 1 & 1 & 1 & 1 & 1 & 1 & 1 & 0 & 0 & 0 & 1 & 8 \\
\hline E3 & 1 & 1 & 1 & 1 & 1 & 0 & 0 & 1 & 0 & 1 & 1 & 8 \\
\hline E4 & 1 & 1 & 1 & 1 & 1 & 0 & 0 & 1 & 0 & 1 & 1 & 8 \\
\hline E5 & 1 & 1 & 0 & 1 & 0 & 0 & 0 & 0 & 1 & 1 & 1 & 5 \\
\hline E6 & 1 & 1 & 1 & 1 & 1 & 0 & 0 & 1 & 0 & 1 & 1 & 8 \\
\hline E7 & 1 & 1 & 1 & 1 & 1 & 1 & 1 & 1 & 1 & 1 & 1 & 11 \\
\hline E8 & 1 & 1 & 1 & 1 & 1 & 1 & 1 & 1 & 1 & 1 & 1 & 11 \\
\hline E9 & 1 & 1 & 1 & 1 & 1 & 1 & 1 & 1 & 1 & 1 & 1 & 11 \\
\hline E10 & 1 & 1 & 0 & 1 & 0 & 0 & 0 & 1 & 0 & 1 & 1 & 6 \\
\hline E11 & 1 & 1 & 0 & 1 & 1 & 0 & 1 & 0 & 0 & 1 & 1 & 7 \\
\hline E12 & 1 & 1 & 0 & 1 & 1 & 0 & 0 & 1 & 0 & 1 & 1 & 7 \\
\hline E13 & 1 & 1 & 0 & 1 & 1 & 0 & 0 & 0 & 1 & 1 & 1 & 7 \\
\hline E14 & 1 & 1 & 1 & 1 & 1 & 0 & 1 & 0 & 1 & 1 & 1 & 9 \\
\hline E15 & 1 & 1 & 0 & 1 & 1 & 0 & 0 & 1 & 1 & 1 & 1 & 8 \\
\hline E16 & 1 & 1 & 0 & 1 & 1 & 0 & 0 & 1 & 0 & 1 & 1 & 7 \\
\hline E17 & 1 & 1 & 0 & 1 & 1 & 0 & 0 & 0 & 0 & 1 & 1 & 6 \\
\hline E18 & 1 & 1 & 0 & 0 & 1 & 0 & 0 & 0 & 1 & 1 & 1 & 6 \\
\hline E19 & 1 & 1 & 1 & 1 & 1 & 0 & 1 & 0 & 1 & 1 & 1 & 9 \\
\hline E20 & 1 & 1 & 1 & 1 & 0 & 0 & 1 & 1 & 0 & 0 & 1 & 7 \\
\hline E21 & 1 & 1 & 1 & 1 & 1 & 1 & 1 & 1 & 1 & 1 & 1 & 11 \\
\hline E22 & 1 & 1 & 1 & 1 & 1 & 1 & 1 & 0 & 0 & 1 & 1 & 9 \\
\hline E23 & 1 & 1 & 1 & 1 & 1 & 0 & 1 & 0 & 0 & 1 & 1 & 8 \\
\hline E24 & 1 & 1 & 1 & 0 & 1 & 1 & 1 & 0 & 0 & 1 & 1 & 8 \\
\hline E25 & 1 & 1 & 1 & 1 & 1 & 0 & 1 & 0 & 0 & 1 & 1 & 8 \\
\hline E26 & 1 & 1 & 1 & 1 & 1 & 0 & 1 & 0 & 0 & 0 & 1 & 7 \\
\hline E27 & 1 & 1 & 1 & 1 & 1 & 0 & 1 & 0 & 0 & 1 & 1 & 8 \\
\hline E28 & 1 & 1 & 1 & 1 & 1 & 0 & 1 & 0 & 0 & 1 & 1 & 8 \\
\hline E29 & 1 & 1 & 0 & 0 & 0 & 1 & 1 & 1 & 1 & 0 & 1 & 7 \\
\hline Total & 29 & 29 & 18 & 26 & 25 & 8 & 17 & 13 & 11 & 25 & 29 & 1 \\
\hline
\end{tabular}

A análise da Tabela 5 também mostra que os estudos incluídos tinham uma estratégia de extração adequada ao contexto da pesquisa (QA5), mas a maioria não conseguiu usar um grupo de controle que pudesse comparar os resultados (QA6). Em 17 estudos os dados foram coletados de forma que abordaram as questões de pesquisa (QA7), entretanto, a análise dos dados foi considera suficientemente rigorosa em 13 estudos (QA7). Já a relação entre o pesquisador e os participantes foi considerada 
adequada em apenas 12 estudos (QA9). Em contrapartida, somente 4 estudos não apresentou uma indicação clara dos resultados (QA10). E por fim, todos os estudos apresentam um valor para pesquisa ou prática (QA11). Quatro dos estudos incluídos na revisão obtiveram a pontuação máxima de 11 pontos [E7, E8, E9 e E21]. A pontuação mais baixa que os artigos alcançaram foi 6 pontos [E1, E10, E17 e E18]. A pontuação média em qualidade dos trabalhos incluídos na revisão é de 7,9.

\subsection{Ameaças a Validade da RSL}

No geral, as principais ameaças à validade da RSL são em relação ao viés de seleção de publicações e aos dados imprecisos extraídos. As pesquisas foram concebidas empregando principalmente recursos eletrônicos. Estes foram pesquisados após a implementação de pesquisas experimentais, strings de busca e utilização de dicionários de sinônimos. Apesar disso, não é possível garantir que todos os estudos relevantes para o tema em discussão foram coletados e há um pequeno risco de que alguns estudos podem ter sido omitidos, devido aos termos de busca utilizados. O processo de extração de dados também pode resulta em dados irrelevantes. Isto pode ocorre, porque o processo de extração de dados foi realizado por apenas um pesquisador. $O$ desenvolvimento de um protocolo da RSL e o uso de uma estratégia de verificação da qualidade da revisão (por um segundo e terceiro revisor) ajuda a garantir que este não era o caso. Finalmente, é possível que os critérios de inclusão podem, ocasionalmente, ter excluídos alguns estudos relevantes. Isso ocorre porque os critérios implementados nos estudos não continham palavras-chaves apresentadas nos títulos ou nos resumos.

\subsection{Trabalhos Relacionados}

Aureliano e Tedesco (2012) desenvolveram e publicaram um estudo sobre o ensinoaprendizagem de programação para iniciantes cujo objetivo foi fornecer uma análise abrangente e sistemática dos trabalhos científicos publicados no Simpósio Brasileiro de Informática na Educação (SBIE) e no Workshop de Informática na Escola (WIE). Para tal, utilizaram uma metodologia de RSL visando extrair, catalogar, analisar e sintetizar dados de todos os artigos publicados em 10 edições (2002-2011) desses eventos.

Da mesma forma, outra pesquisa mapeou um cenário mais específico dentro da temática deste trabalho. Medeiros, Silva e Aranha (2013) apresentou uma RSL para o ensino e aprendizagem de programação com jogos digitais no contexto nacional. Este estudo desenvolveu um processo similar ao apresentado por Medeiros, Silva e Aranha (2013), aplicando a técnica de pesquisa secundária (a RSL) para realizar uma análise abrangente e sistemática da pesquisa em jogos digitais para o ensino e aprendizagem de programação no cenário internacional.

\section{Resultados Gerais}

Nesta seção, apresentam-se as respostas às questões de pesquisa desta RSL. Um resumo das informações que foram extraídos de cada estudo incluído pode ser encontrado em: $<$ http://goo.gl/jnzr3o>.

\subsection{Resposta a Questão de Pesquisa (QP1)}

Ao analisar os estudos incluídos na RSL, foram encontrados 7 tipos diferentes de linguagens de programação utilizadas. Java foi a principal linguagem de programação 
empregada, sendo encontrada em 8 estudos [E1, E6, E9, E13, E14, E15, E22 e E29]. Seguido por 3 artigos que aborda o uso da linguagem C\# [E8, E18 e E24]. Visual Basic [E8 e E23], Python [E10 e E17] e C++ [E7 e E26] foram reportados em 2 artigos cada. $\mathrm{C}$ [E27] e F\# [E8] foram descritos por um artigo respectivamente. Os demais estudos não apresentaram as linguagens de programação utilizadas. Os estudos destacam que também foram utilizados softwares concebidos para ensinar princípios de programação. Isso inclui o uso do Greenfoot [E9, E13, E15, E17 e E28], Alice [E1, E13, E14 e E16], Scratch [E16 e E25], Blocky [E3] e GameMaker [E29].

A utilização da Robótica para ensinar princípios de programação também foi reportada. O uso do Lego Mindstorms utilizada para função lúdica e didática abordando a teoria e a prática de conteúdos direcionados para a introdução de programação com o uso da robótica foi empregada em dois estudos [E2 e E3]. A programação de Robôs como o LighBot, um ambiente de programação educacional simples que permite que os iniciantes aprendam as noções básicas de programação de um robô simulado, foi destacado em [E19]. Em [E10], é apresentado um jogo chamado Gidget, em que os alunos jogam através de uma série de níveis, encontrando e corrigindo defeitos em um robô.

\subsection{Resposta a Questão de Pesquisa (QP2)}

Nos estudos reportados, a utilização de estudo de caso foi o método mais empregado. Dez estudos relatam o uso desta metodologia, correspondendo a 35\% do total de estudos [E6, E7, E8, E9, E13, E16, E17, E18, E19 e E21]. A utilização de experimentos controlados foi reportada em seis estudos [E3, E10, E12, E20, E25, E26], correspondendo a 21\%. Relato de experiência foi empregado em quatro artigos [E5, E14, E22 e E29], perfazendo um total de 14\%. A utilização de oficinas foi reportada em três estudos [E15, E27 e E28], correspondendo a um total de 10\%. Quase experimento [E4 e E23] e Questionários [E11 e E24] foram empregados em dois estudos, completando um total de 14\%. Além disso, a Avaliação Experimental [E2] e Entrevistas [E1] também ocorreram, correspondendo a um total de 6\%. A Figura 1(a) ilustra a porcentagem de cada estudo empregado. Esses dados mostram que em 18 estudos foram realizados utilizando algum método empírico de pesquisa (estudo caso, experimento controlado e quase experimento) e 11 estudos não foi utilizado nenhum método empírico de pesquisa. É ilustrada na Figura 1(b) a porcentagem desses dados.

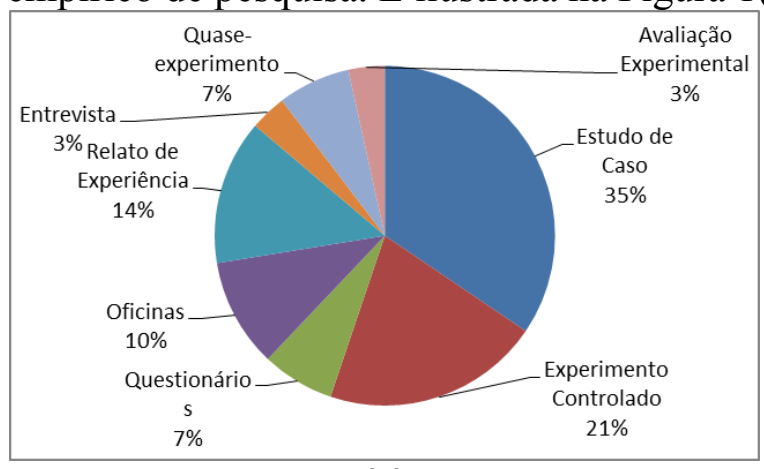

(a)

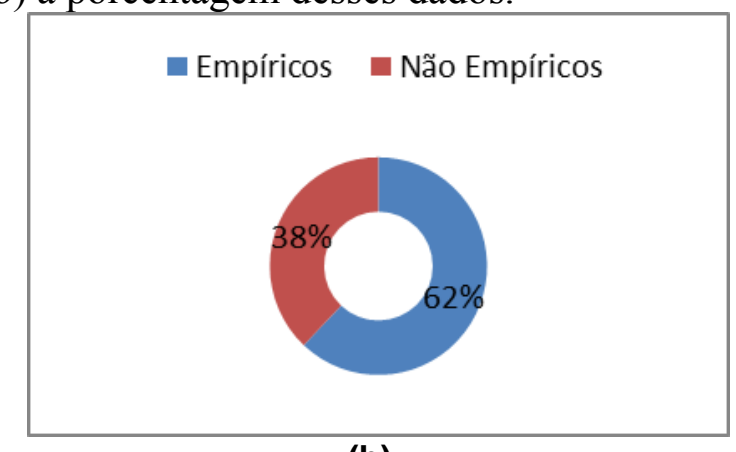

(b)

Figura 1: (a) Tipos de estudos reportados na RSL e (b) métodos de pesquisa empregados.

\subsection{Resposta a Questão de Pesquisa (QP3)}


A escala de estudos incluídos na revisão variou muito. Estes variam de estudos de pequena escala que continham $8,9,10$ e 13 participantes, a estudos de escalas maiores, que informou sobre o tamanho das amostras. Teve estudos que participarem 100, 146, 233, 322 e a maior amostra foi de 325 participantes. 27 trabalhos relatam o número exato de alunos que participaram da pesquisa realizada. Em contrapartida, dois estudos [E6 e E22] não declararam o número de participantes envolvidos.

\subsection{Resposta a Questão de Pesquisa (QP4)}

Depois de analisar todos os estudos incluídos na RSL, é possível apresentar uma classificação sobre se a literatura incluída relata o uso de jogos digitais como um método eficaz ao ensinar e aprendizagem de programação. Dos 29 artigos incluídos em 28 (97\%) relatam que o uso de jogo é eficaz ao ensinar conceitos de programação, e somente um estudo oferece resultado misto [E14], ou seja, pode trazer resultados positivos e negativos, mas esse mesmo estudo não relatou os pontos negativos. Nenhum estudo relata que o uso é considerado ineficiente para ensinar programação.

\subsection{Resposta a Questão de Pesquisa (QP5)}

Os artigos foram classificados de acordo com o nível de escolaridade para os quais as pesquisas se destinaram. Observando a Figura 2 é possível perceber que a maioria dos estudos são voltados para o ensino superior (55\%) [E1, E2, E4, E5, E8, E10, E11, E12, E19, E20, E21, E22, E23, E24, E26 e E29]. Em contraste 39\% dos estudos analisados tem como foco o ensino médio [E6, E7, E8, E9, E13, E14, E15, E16, E17, E18, E25 e E28]. Apenas 3\% das pesquisas destinam-se ao ensino fundamental [E27]. Esta revisão ainda apresenta que $3 \%$ dos artigos destinam ao ensino em vários níveis, tanto no médio, fundamental, como superior [E3]. Esses dados apresentam que 43\% dos estudos estão sendo destinados ao ensino básico (fundamental e médio) e $57 \%$ ao ensino superior, mostrando que o ensino de programação no nível básico é uma tendência que vem sendo empregadas pelos pesquisadores em suas pesquisas.

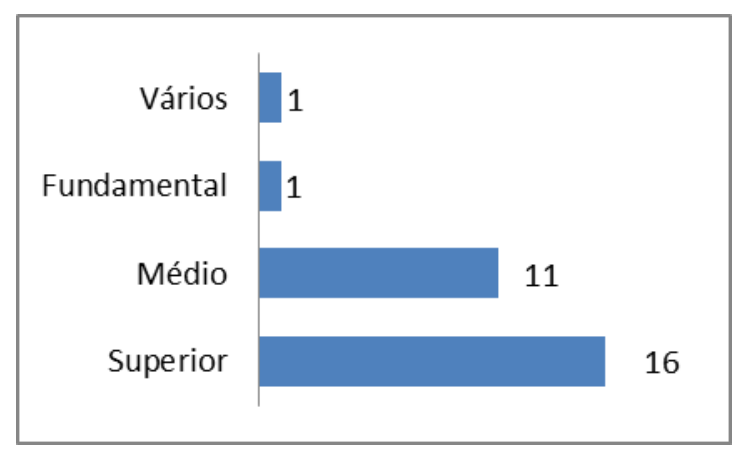

Figura 2: Estudos classificados pelo nível educacional.

\section{Conclusões}

Nesta pesquisa, apresentamos os resultados de uma RSL sobre o processo de ensino e aprendizagem de programação através de jogos digitais publicados nos últimos cinco anos, em relevantes eventos e periódico na área no cenário internacional. A busca realizada nesta revisão resultou na pré-seleção de 95 estudos, dentre os quais 29 foram incluídos para a extração de dados. Os jogos digitais para o ensino e aprendizagem de programação estão voltados para melhorar o rendimento, interesse dos alunos em 
disciplinas de programação e segundo os resultados da RSL, esse método ajuda o desempenho dos alunos, diminui as desistências e aumenta a motivação.

Neste contexto, várias observações podem ser feitas diante do resultado desta revisão. Em relação ao índice de qualidade, acreditamos que este número é bom (com uma média de 7.9/11). Isto é devido a 18 dos 29 artigos incluídos na revisão aplicarem algum método de estudo empírico. Esses estudos pontuam bem em relação aos critérios de avaliação da qualidade utilizados.

Cinco questões de pesquisa foram criadas a fim de determinar a eficácia de usar jogos no ensino e aprendizagem de programação e para fornecer uma visão ampla da temática da pesquisa. Vários resultados e tendências, no que diz respeito ao ensino e aprendizagem de programação usando jogos, poderão ser indicados com resultado. Estes incluem as observações de que, (i) a linguagem de programação Java é a que tem sido mais frequentemente adotada nas pesquisas; (ii) estudos de casos e experimentos controlados são os métodos mais comumente relatados e utilizados para avaliar a eficácia da intervenção dos jogos; (iii) o número de participantes que tomaram parte na pesquisa para avaliar a eficácia do uso de jogos no ensino de programação varia muito de estudo para estudo; e (iv) metade das pesquisas está sendo aplicadas ao ensino superior.

Os estudos primários analisados são uma importante fonte de informações sobre as diversas mudanças enfrentadas nessa área ao longo dos anos. Uma das observações mais importantes percebidas foi à aplicação de métodos empíricos como meio de avaliação dos resultados obtidos pelas pesquisas desenvolvidas. Os estudos reportam que em $62 \%$ foi aplicado algum método empírico de pesquisa. Este dado confronta com os apresentados em Medeiros, Silva e Aranha (2013) em que as pesquisas nacionais na área, são pouco os estudos em que foram aplicados algum método de pesquisa empírico. Todos, sem exceção, utilizaram mecanismos que ensinam programação criando ou propondo um jogo. Com base neste cenário, pode-se notar que há necessidade de que a comunidade científica nacional reúna esforços no que tange à elaboração de trabalhos que visem a avaliar as abordagens propostas de modo mais efetivo, por meio de estudos de caso e/ou experimentos controlados. Apesar dos resultados apresentados serem considerados relevantes.

As publicações classificadas aqui como não empíricas representam as pesquisas de caráter fundamentalmente descritivo, como a apresentação relato de experiência, entrevistas, oficinas e aplicação de questionários. Esta pesquisa representa $38 \%$ dos estudos encontrados.

Por questões de espaço, outras conclusões realizadas sobre os dados coletados não puderam ser apresentadas neste artigo. Uma versão ampliada deste artigo poderia apresentar estes resultados. No geral, os resultados da RSL indicam que a utilização de jogos é uma ferramenta eficaz para ensinar programação. Isto é verificado em $97 \%$ dos estudos.

\section{Agradecimentos}

Os autores agradecem a Coordenação de Aperfeiçoamento de Pessoal de Nível Superior (CAPES) pelo suporte parcial a esta pesquisa, ao Instituto Nacional de Engenharia de 
Software (INES), ao Conselho Nacional de Desenvolvimento Científico e Tecnológico (CNPq), ao PROEXT - MEC/SESu, e em especial ao Programa de Pós-Graduação em Sistemas Computacionais - PPgSC/UFRN, por toda infraestrutura oferecida.

\section{Referências}

Adamo-Villani, N.; Haley-Hermiz, T.; Cutler, R. (2013) "Using a Serious Game Approach to Teach 'Operator Precedence' to Introductory Programming Students”. In: International Conference Information Visualisation - IV, p.523-526.

Aureliano, V. C. O.; Tedesco, P. C. A. R. (2012) "Ensino-aprendizagem de Programação para Iniciantes: uma Revisão Sistemática da Literatura focada no SBIE e WIE”. In: XXIII Simpósio Brasileiro de Informática na Educação - SBIE.

Brereton, P.; Kitchenhama, B. A.; Budgenb, D.; Turnera, M.; Khalilc, M. (2007) "Lessons from applying the systematic literature review process within the software engineering domain". In: Journal of Systems and Software 80 (4) 571-583.

Dybå, T.; Dingsøyr, T. (2008) "Empirical Studies of Agile Software Development: A Systematic Review". In: Information and Software Technology, v.51, p. 833-859.

Kitchenham, B.; Brereton, P.; Budgen, D.; Turner, M.; Bailey, J.; Linkman, S. (2009) "Systematic literature reviews in software engineering - A systematic literature review". In: Information and Software Technology, v.51, p.7-15.

Kyriacou, M. T.; Brereton, O. P. (2011) "Systematic Literature Review: Teaching Novices Programming Using Robots". In: International Conference on Evaluation and Assessment in Software Engineering - EASE.

Major, L., Kyriacou, T., Brereton, O. P. (2011) "Systematic Literature Review: Teaching Novices Programming Using Robots". In: International Conference on Evaluation and Assessment in Software Engineering - EASE.

Marques, D. L.; Costa, L. F. S.; Silva, M. A. A.; Rebouças, A. D. D. S. (2011) "Atraindo Alunos do Ensino Médio para a Computação: Uma Experiência Prática de Introdução a Programação utilizando Jogos e Python”. In: Simpósio Brasileiro de Informática na Educação - SBIE, Aracaju - SE.

Medeiros, T. J.; Silva, T. R.; Aranha, E. H. S. (2013) "Ensino de programação utilizando jogos digitais: uma revisão sistemática da literatura". In: Revista Novas Tecnologias na Educação - RENOTE, v.11, n.3.

Raabe, A. L. A.; Silva, J. M. C. (2005) "Um ambiente para atendimento as dificuldades de aprendizagem de algoritmos". In: XIII Workshop de Educação em Computação WEI, p.2326-2335.

Rebouças, A. D. D. S.; Marques, D. L.; Costa, L. F. S.; Silva, M. A. A. (2010) "Aprendendo a Ensinar Programação Combinando Jogos e Python". In: XXI Simpósio Brasileiro de Informática na Educação - SBIE.

Sá, E. J. V; Teixeira, J. S. F; Fernandes, C. T. (2007) "Design de atividades de aprendizagem que usam Jogos como princípio para Cooperação". In: XVIII Simpósio Brasileiro de Informática na Educação - SBIE. 